OPEN ACCESS

Edited by:

Terence Y. Pang,

Florey Institute of Neuroscience and

Mental Health, Australia

Reviewed by:

Wenyan Cui,

Zhejiang University, China

Julia L. Drewes,

Johns Hopkins University School of

Medicine, USA

*Correspondence:

Dietmar Fuchs,

Division of Biological Chemistry, Biocenter, Medical University of

Innsbruck, Innrain 80, Innsbruck

6020, Austria

dietmar.fuchs@i-med.ac.at

Specialty section:

This article was submitted to Molecular Psychiatry, a section of the journal Frontiers in Psychiatry

Received: 29 November 2014 Accepted: 17 June 2015

Published: 14 July 2015

Citation:

Gostner JM, Becker K, Kurz K and

Fuchs D (2015) Disturbed amino acid

metabolism in HIV: association with neuropsychiatric symptoms.

Front. Psychiatry 6:97.

doi: 10.3389/fpsyt.2015.00097

\section{Disturbed amino acid metabolism in HIV: association with neuropsychiatric symptoms}

\author{
Johanna M. Gostner ${ }^{1}$, Kathrin Becker ${ }^{2}$, Katharina Kurz ${ }^{3}$ and Dietmar Fuchs ${ }^{2 *}$ \\ ${ }^{1}$ Division of Medical Biochemistry, Biocenter, Medical University of Innsbruck, Innsbruck, Austria, ${ }^{2}$ Division of Biological \\ Chemistry, Biocenter, Medical University of Innsbruck, Innsbruck, Austria, ${ }^{3}$ Department of Internal Medicine VI, Medical \\ University of Innsbruck, Innsbruck, Austria
}

Blood levels of the amino acid phenylalanine, as well as of the tryptophan breakdown product kynurenine, are found to be elevated in human immunodeficiency virus type 1 (HIV-1)-infected patients. Both essential amino acids, tryptophan and phenylalanine, are important precursor molecules for neurotransmitter biosynthesis. Thus, dysregulated amino acid metabolism may be related to disease-associated neuropsychiatric symptoms, such as development of depression, fatigue, and cognitive impairment. Increased phenylalanine/tyrosine and kynurenine/tryptophan ratios are associated with immune activation in patients with HIV-1 infection and decrease upon effective antiretroviral therapy. Recent large-scale metabolic studies have confirmed the crucial involvement of tryptophan and phenylalanine metabolism in HIV-associated disease. Herein, we summarize the current status of the role of tryptophan and phenylalanine metabolism in HIV disease and discuss how inflammatory stress-associated dysregulation of amino acid metabolism may be part of the pathophysiology of common HIV-associated neuropsychiatric conditions.

Keywords: HIV, tryptophan, kynurenine, indoleamine 2,3-dioxygenase, phenylalanine, tyrosine, phenylalanine 4-hydroxylase, tetrahydrobiopterin

\section{Introduction}

Human immunodeficiency virus type 1 (HIV-1) infection is associated with neuropsychopathologic disturbances that range from behavioral changes and mild cognitive and motor impairments to depression and severe mental problems as reported in patients with manifested AIDS dementia complex (1). These conditions are comprehensively termed HIV-associated neurocognitive disorders (HAND). Besides the presence and severity of neurocognitive impairment and functional decline, the definitional criteria for HAND also comprise a variety of neuropsychiatric relevant comorbid conditions (2). Steady deterioration of neurocognitive performance in patients not only impairs their quality of life but is also associated with an increased mortality rate. Although it has been reported that effective antiretroviral therapy (ART) can partially preserve or even improve neurological function and decrease morbidity and mortality (3), neurocognitive disturbances are still highly prevalent in HIV patients receiving treatment (4). The development of depressive symptoms is the most frequent manifestation (5-8). A number of psychosocial aspects, in addition to pathophysiological factors, may contribute to the high rate of depressive illness in these patients (7).

The molecular basis of HAND- and HIV-associated depressive symptoms still remains to be elucidated in detail (9). The incomplete clearance of viral load owing to the poor accessibility of the central nervous system to antiretroviral drugs or the development of resistant virus strains during therapy is suspected to be of importance (10). In addition, neuronal damage 
may arise from toxic viral products or from activated brain macrophages and microglia releasing inflammatory mediators $(9,11)$. Increasing evidence suggests that changes in amino acid metabolism occur as a result of these viral and inflammatory insults during infection, and that these metabolic changes may play a critical role in HAND. This review will cover recent advances in the field of inflammatory stress-associated dysregulation of amino acid metabolism in HIV and its association with neuropsychiatric symptoms.

\section{Metabolic Changes in HIV Disease}

HIV disease is characterized by severe metabolic changes. Anorexia, malabsorption, increased resting energy expenditure, and specific disturbances in protein turnover are common in HIV and AIDS patients $(12,13)$. However, not only HIV infection itself but also effective treatment is associated with the development of severe metabolic disorders, such as insulin resistance, diabetes mellitus (14), and lipodystrophy syndrome (15).

The immune response and metabolic pathways are highly cross-regulated (16). Also, depression and cognitive impairment are closely linked to chronic inflammation. Changes in amino acid metabolism and neurotransmitter synthesis play a major role in the etiology of such conditions $(17,18)$ and constitute potential intervention points for therapeutic strategies. In this regard, phenylalanine (Phe) and tryptophan (Trp) metabolism are among the most intensively discussed pathways in the literature $(18,19)$.

Diminished breakdown of Phe to tyrosine (Tyr) (20) and the accelerated conversion of Trp to kynurenine (Kyn) (21) were shown to correlate with elevated levels of immune activation markers, e.g., neopterin or interferon- $\gamma$ (IFN- $\gamma$ ), in HIV-infected individuals $(22,23)$. Trp and Tyr are precursor molecules for serotonin and dopaminergic neurotransmitters, respectively. Thus, disturbed metabolism can negatively affect neuropsychoimmunological circuits (24) and can contribute to the pathophysiology of common HIV-associated neuropsychiatric symptoms.

Phenylalanine is converted to Tyr via the tetrahydrobiopterin $\left(\mathrm{BH}_{4}\right)$-dependent enzyme phenylalanine 4-hydroxylase. Phe turnover is reduced in chronic inflammatory conditions, consequently affecting dopamine, adrenaline, and noradrenaline synthesis, as tyrosine is the precursor of these neurotransmitters. Also, maintenance of adequate levels of tryptophan is essential, as tryptophan hydroxylation via the $\mathrm{BH}_{4}$-dependent enzyme tryptophan 5-hydroxylase is the rate limiting step for 5-hydroxytryptamine (serotonin) formation. During inflammation, peripheral Trp levels are predominantly dependent on the activity of enzyme (IDO1), which is activated mainly via the T helper (Th) type 1 cytokine IFN- $\gamma$.

In the following, relevant inflammatory stress-related pathways (Figure 1) will be introduced followed by describing their alteration in HIV disease in patients on and off therapy.

\section{Tryptophan Metabolism}

Breakdown of Trp into Kyn is a crucial biochemical pathway within the biosynthesis of nicotinamide adenosine dinucleotides $(\mathrm{NAD} / \mathrm{NADH})$ that are required cofactors for many enzymes.
The pathway is also important for the functioning of immune responses because during immune activation, Th1 cytokines, most importantly, IFN- $\gamma$, induce the expression and activation of enzyme IDO1 (EC:1.13.11.52) in human monocytes/macrophages and dendritic cells (25). In addition, other cell types, such as endothelial, epithelial cells, and fibroblasts, are able to activate IDO1 in response to inflammatory stimuli. Deprivation of this essential amino acid not only inhibits pathogen growth, but also slows down $\mathrm{T}$-cell responses, thereby being a regulatory feedback mechanism that protects the immune system from overreactions (26). IDO1 activation is associated with the generation of a regulatory phenotype in T cells and dendritic cells $(27,28)$, and is involved in tumor immune escape (29). Chronic immune stimulation and consecutive IDO activation might also facilitate HIV persistence by inducing T cell apoptosis and tolerance.

IDO1 is the rate-limiting enzyme in the conversion of Trp to Kyn, and thus the Kyn/Trp ratio can be used to estimate IDO1 activity $(21,30)$. Accelerated Trp breakdown has been reported for several diseases associated with chronic immune activation such as infection, autoimmune syndromes, malignancies, and neurodegenerative disorders, as well as cardiovascular diseases (31). Already in the 90s, accelerated Trp breakdown was shown to correlate with elevated levels of IFN- $\gamma$ and the immune activation marker neopterin in HIV patients $(21-23,32)$, and an inflammatory stress-related $\operatorname{Trp}$ decrease was suggested to be involved in the development of neuropsychiatric symptoms (33). In 2008, another study demonstrated that HIV-infected patients with depression presented with higher plasma neopterin concentrations, a higher degree of tryptophan degradation, and lower quality of life scores than non-depressive patients (34). Interestingly, correlations between enhanced depression and quality of life scores on the one hand, and tryptophan degradation and neopterin levels on the other hand, were only found in patients without antidepressant medication. In line with these findings, Martinez and colleagues reported recently that depression severity in HIV patients was associated with a decrease in plasma Trp concentration and an increase in Kyn/Trp. In the 12-month follow-up study in a cohort of 504 patients, ART was able to partially reduce Trp breakdown, which went along with improvement of depressive symptoms (35).

\section{Downstream Metabolites of the Trp-Kyn Pathway}

In addition to the depletion of Trp, Kyn downstream products may be involved in immunoregulation and in the pathogenesis of depression. Kyn itself is an endogenous ligand of the arylhydrocarbon receptor and activation of arylhydrocarbon signaling is involved in T-cell differentiation and the development of tolerance (36, 37). 3-hydroxyanthranilic (3-HAA) and quinolinic acid (QA) were shown to selectively induce apoptosis in mouse thymocytes as well as in Th1, but not Th2, cells in vitro $(38,39)$.

Furthermore, a number of kynurenine breakdown products have been reported to be neuroactive. Deregulated production of 3-HAA, 3-hydroxykynurenine (3-HK), and QA has been observed in several neurologic and psychiatric disorders (40). 


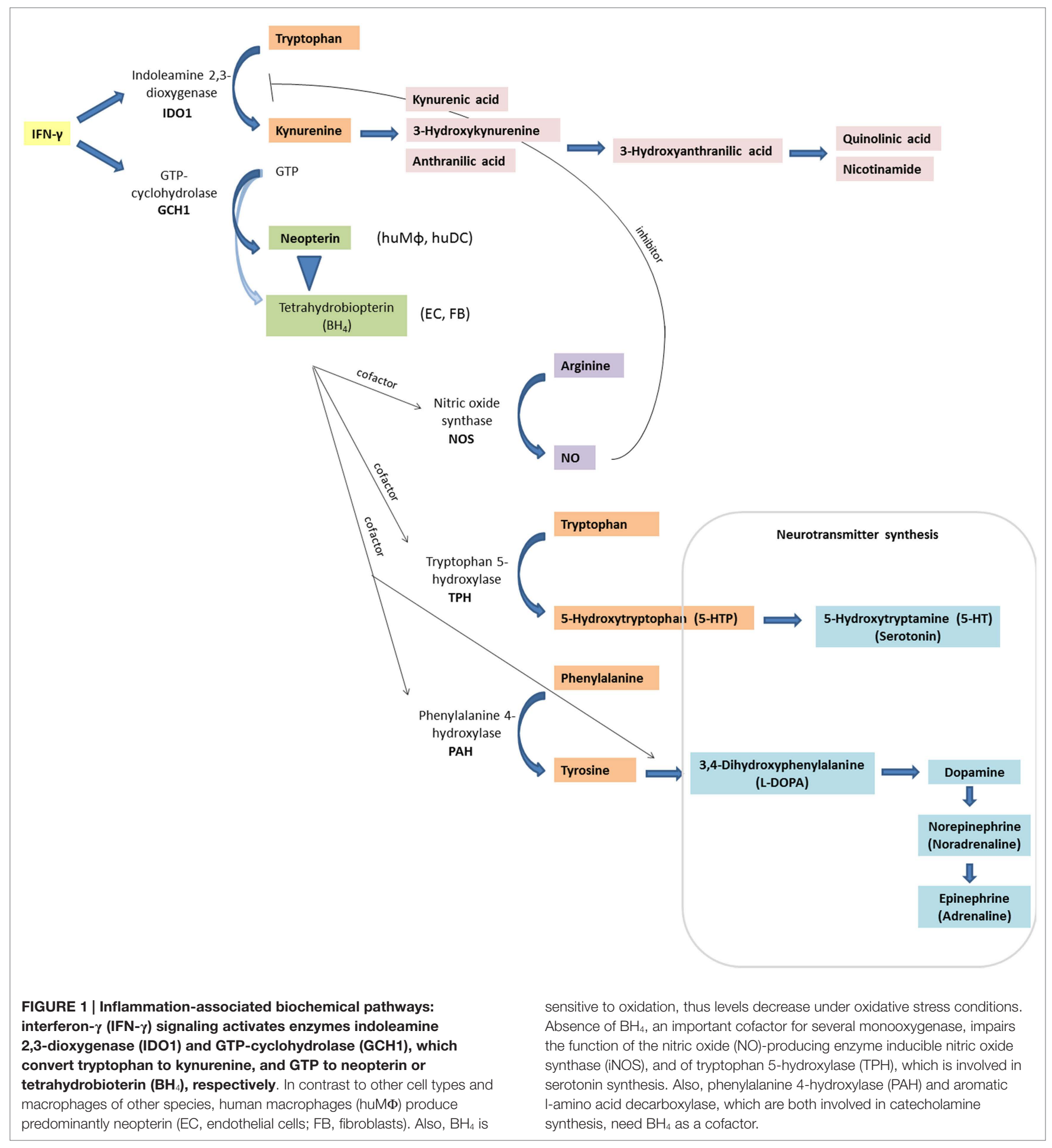

While some metabolites such as kynurenic acid (KA) can be neuroprotective (41-43), QA released by activated macrophages was shown to exert excitotoxicity (44), thereby contributing to brain injury [see also review by Kandanearatchi and Brew (45)]. When submicromolar concentrations of QA were infused for a longer period into the brain of rats, neuronal loss occurred preferentially in the striatum and the hippocampus - the regions that are involved in HAND neuropathologically (46).

Elevated QA levels were shown in brain tissue of patients with HIV-1-associated dementia $(47,48)$, and cerebrospinal fluid (CSF) levels of QA were shown to increase during HIV infection and to correlate with HAND severity (49-51). Also, in macaques 
infected with simian immunodeficiency virus (SIV), the severity of neurological symptoms was related to their CSF QA levels (41). Of note, the HIV-1 proteins Nef and Tat are also able to induce the synthesis of QA by macrophages (52). In a mouse model of peripheral immune activation, which was induced by bacille Calmette-Guerin, IDO activation and upregulation of the quinolinic acid synthesizing enzyme 3-hydroxyanthranilic acid oxygenase (3-HAO) in the brain was shown to play a key role in the development of depressive-like behavior in mice (53). Similarly, a single intracerebroventricular injection of the HIV-1 protein Tat induced depressive-like behavior in exposed mice, and IDO expression was demonstrated in the CNS tissue of the same mice 4 and $24 \mathrm{~h}$ after treatment (54). In a recent study on longitudinal changes of Trp-Kyn metabolism in the brains of SIV-infected macaques, Drewes and colleagues confirmed the predictive potential of QA/Trp ratios for CNS associated symptoms (55). Further, while effective combination ART was able to stabilize Trp metabolite concentrations in the CSF of most of these animals, levels could not be restored in the striatum, indicating the only partial effectiveness of the therapy. Interestingly, inhibition of QA formation by 6-chloro-D-tryptophan in HIV-infected macrophages was demonstrated to prevent neuronal damage in human fetal brain aggregate cultures (56).

Furthermore, increased 3-HK levels and elevated kynureninase (KYNU) activity in the frontal cortex of HIV-infected individuals have also been reported, whereby levels were increased in both non-demented and demented HIV patients compared to controls, with the increases being more pronounced for the latter (57).

Accelerated tryptophan breakdown in the periphery and neuropsychiatric changes in the brain are closely linked as brain Kyn metabolite levels are influenced by fluctuations in the concentrations of circulating Trp, Kyn, and 3-HK, which can cross the blood-brain barrier (58). Also, microglial cells and bloodborne cells in the brain can be stimulated to activate the Trp-Kyn pathway during peripheral immune activation (40). Interestingly, IDO activity was significantly increased in the frontal cortex of post-mortem brains of demented HIV-patients compared to age-matched controls, while the activity was only slightly but not significantly elevated in non-demented HIV-patients (57). This finding was consistent with the report of increased enzyme activity in the cerebral cortex of retrovirus-infected macaques (59).

Taken together, the above studies strongly indicate that IDO activation and consecutive accumulation of neurotoxic metabolites play an important role in the development of neurocognitive disturbances. Accordingly, targeting IDO or p38 MAPK expression was proposed by $\mathrm{Fu}$ and coworkers as a promising therapy approach to prevent/treat comorbid depressive disorders in HIV1 -infected patients (60).

However, although the conversion to Kyn is the major breakdown route of Trp, accounting for about $90 \%$ of Trp metabolism $(61,62)$, there are also alternative pathways that do not involve the oxidative cleavage of the indole ring, such as the formation of the neurotransmitter serotonin (5-hydroxytryptamine, 5-HT) via tryptophan 5-hydroxylase (TPH, EC:1.14.16.4). Thus, low Trp availability due to persistent immune activation is linked to impaired synthesis of serotonin and can contribute to neurotransmitter imbalance. Indeed, CSF levels of serotonin were found to be significantly lower in a group of 21 asymptomatic, early disease stage HIV-positive patients compared to healthy controls (63). In this study, no correlation was found for 5-HT levels and CD4 cell count or depressive behavior, which could be due to the early stage of disease. In the SIV-infected macaque model, striatal serotonin concentrations decreased during acute and chronic infection and could be partially restored by combined ART, while no correlation of serotonin levels with Trp-Kyn pathway activation marker QA/ Trp or encephalitis severity could be observed (55).

The antidepressant paroxetine, which elevates serotonin availability, has previously been applied in different clinical settings with the aim to improve mood and cognitive symptoms (64). Results from patients on IFN- $\alpha$ therapy suggested that cell-mediated immunity and Trp-Kyn pathway activation is not affected by the drug (17). Recently, a combination of fluconazole and paroxetine were shown to be potentially neuroprotective in the SIV-infected macaque model of HIV-associated CNS disease (65). However, although markers of neurodegeneration were decreased in the frontal cortex, serotonin levels could not be significantly elevated and circulating markers of neuroinflammatory origin were not affected. Also, other psychiatric medications are frequently used as adjunct therapies for HIV-associated psychiatric comorbidities and HAND; and trial data on depression and psychosis treatments in HIV-infected patients reported a beneficial effect of selective serotonin reuptake inhibitors (SSRIs) and tricyclic antidepressants (66). However, there are concerns with respect to adverse effects such as worsening of metabolic syndrome when combining antidepressant/antipsychiotic therapies with antiretrovirals (67).

\section{Vitamin Status, Nutrition, and Inflammatory Stress}

Inflammatory disorders are characterized by oxidative stress conditions, which lead to low plasma levels of antioxidants, thus also of vitamins. Nutritional imbalances and deficiencies may contribute to neuropsychiatric disturbances and immune dysregulation associated with HIV-1 infection (68).

For example, low vitamin $\mathrm{B}_{6}$ status is related to altered neuropsychiatric function, and the normalization of vitamin $\mathrm{B}_{6}$ status in HIV-infected patients was found to be associated with a decline in psychological distress (69). High Trp turnover leads to an increased demand for vitamin $\mathrm{B}_{6}$ for downstream processing. Enzymes of the Trp-Kyn pathway that use vitamin $B_{6}$ (pyridoxal $5^{\prime}$-phosphate, PLP) as a cofactor are KYNU, which converts Kyn to anthranilic acid (AA), and 3-HK to 3-HAA, and kynurenine aminotransferases (KAT), which convert Kyn to KA and 3-HK to xanthurenic acid (70). Low vitamin $\mathrm{B}_{6}$ status is associated with altered excretion of tryptophan metabolites $(71,72)$. Also, aromatic-L-amino-acid decarboxylase (AADC), which catalyzes the decarboxylation of L-3,4-dihydroxyphenylalanine (L-DOPA) to dopamine and of 5-hydroxytryptophan (5-HPT) to serotonin, uses PLP as cofactor (73).

Several approaches to influence the synthesis of serotonin and catecholamine neurotransmitters via administration of the appropriate precursor amino acids have been reported (74-76). However, regarding a direct supplementation of tryptophan, it 
should be considered that there is a risk of elevating concentrations of neurotoxic catabolites (77). Moreover, selective supplementation unlikely results in long-term influence on immunopathogenesis, and thus on the clinical course of cognitive disorders. A multitude of oxidative sensitive molecules are simultaneously affected by inflammatory stress, leading to lower levels of plasma antioxidants $(78,79)$. Otherwise, more complex dietary interventions might bear a great potential to increase the clinical benefits of HIV patients. Certainly, nutritional and metabolic parameters play an important role in the pathophysiology of HIV infection and might have therapeutic implications $(13,68)$, which is particularly relevant for individuals suffering from severe nutritional deficiencies (80). For example, in a study cohort of 504 $\mathrm{HIV}$-infected patients undergoing first ART, low protein nutrition was associated with severity of depression (35). It should also be mentioned that about $95 \%$ of the body's serotonin resides in the gut (81) and a number of food-derived antioxidants have been shown to suppress IDO1 activity in vitro $(82,83)$.

In addition to IDO1, hepatic enzyme tryptophan 2,3-dioxygenase (TDO2, EC 1.13.11.11) is able to metabolize Trp. TDO2 activity is regulated by tryptophan levels and by glucocorticoids $(84,85)$. To relate increased Trp breakdown rates to inflammation-induced IDO1, the concomitant estimation of pro-inflammatory molecules is required. Thereby, the pteridine neopterin has turned out to be a reliable biomarker for inflammation-induced oxidative stress (86).

\section{Neopterin/Biopterin Metabolism and Inflammatory Stress}

Activation of both IDO1 and GCH1 is highly responsive to IFN- $\gamma$ signaling (87). Neopterin levels are increased in several disorders associated with chronic immune activation (86). In addition, elevated neopterin concentrations are predictive for the development of AIDS and survival in HIV-1 seropositives $(23,33)$. Also, higher neopterin was shown to correlate with lower levels of plasma antioxidants (79). Of note, the peripheral monocytes of patients with HIV-associated dementia were shown to express lower levels of the antioxidant protein thioredoxin, as well as of enzymes peroxiredoxin and sodium oxide dismutase compared to patients with normal cognition (88). These altered functions of immune cells and the decreased expression of antioxidant defense systems may contribute to inflammation-induced neuronal damage.

Neopterin is formed by the enzyme GTP-cyclohydrolase (GCH1, EC 3.5.4.16). GCH1 converts guanosine-5' -triphosphate (GTP) to 7,8-dihydroneopterintriphosphate, the precursor of neopterin, 7,8-dihydroneopterin, and 5,6,7,8-tetrahydrobiopterin $\left(\mathrm{BH}_{4}\right)(86)$. Most cell types predominantly produce $\mathrm{BH}_{4}$ upon activation of GCH1 and lower amounts of 7,8-dihydroneopterin and neopterin. $\mathrm{BH}_{4}$ is highly sensitive to oxidation and its concentrations rapidly decrease under oxidative stress conditions $(89,90)$. In addition, human monocyte-derived macrophages lack sufficient 6-pyruvoyltetrahydropterin synthase (PTPS, EC 4.2.3.12), the enzyme responsible for the formation of $\mathrm{BH}_{4}$ from 7,8-dihydroneopterintriphosphate. Therefore, these cells produce large amounts of 7,8-dihydroneopterin/ neopterin in a relatively constant ratio of 3:1 (91-93).
$\mathrm{BH}_{4}$ is an important cofactor for several monooxygenases including nitric oxide synthase (NOS) (Figure 1). Lack of $\mathrm{BH}_{4}$ formation by activated human macrophages may be one reason for the low activity of inducible NOS (iNOS) in immune cells and the generally lower nitrite/nitrate levels in human plasma compared with those in rodents (94).

However, NOS function in other cell types might not be affected in this extent. Interestingly, an increase of serum nitrate in HIV patients has been reported; however, statistical significance of changes differed among these studies depending on disease stage and viral replication and presence of opportunistic infection (95-97). Boven et al. reported elevated levels of nitrotyrosine, indicating the increased formation of peroxynitrite, in brain sections of demented HIV patients compared with non-demented patients (98). The origin of these NOx species is yet unknown. An involvement of neuronal (nNOS) or endothelial NOS (eNOS) in nitric oxide (NO) production could play a role. Importantly, endothelial dysfunction and increased risk of cardiovascular disease are frequently associated with HIV-disease and therapy $(99,100)$. Of note, NO was shown to strongly inhibit IDO1 activity (101). Both the origin and the functional consequences of changing NO levels in HIV disease have yet to be investigated in more detail.

\section{$\mathrm{BH}_{4}$ Deficiency, Phenylalanine Metabolism, and Catecholamine Synthesis}

In addition to being required for $\mathrm{NOS}$ activity, $\mathrm{BH}_{4}$ is also cofactor for the enzyme phenylalanine 4-hydroxylase (PAH, EC:1.14.16.1). Insufficient cofactor availability impairs the functioning of $\mathrm{PAH}$, resulting in reduced production of catecholamine neurotransmitters dopamine, norepinephrine (noradrenaline), and epinephrine (adrenaline). The precursor amino acid for catecholamines is Tyr, which is formed from Phe by PAH. Also, the conversion of Tyr to L-DOPA via tyrosine 3-monooxygenase (alternative name: tyrosine 3-hydroxylase, $\mathrm{TH}$ ) requires $\mathrm{BH}_{4}$ as cofactor (92).

Dysregulation of Phe metabolism was found in patients with cancer, patients with multiple trauma with sepsis, and in the elderly, as well as in patients with HIV-1 infection (19, 20, 102-104). Plasma Phe/Tyr ratio measured in HIV-1-infected individuals was found to be increased and to correlate with the concentrations of the immune activation marker neopterin, as well as with HIV-RNA levels and CD4+ counts (20). In the same study, it was shown that effective ART could reduce plasma concentrations of Phe and neopterin. The Phe/Tyr ratio is a convenient way to determine $\mathrm{PAH}$ activity in the absence of a reliable methodology for $\mathrm{BH}_{4}$ measurements (105). Thus, the increase of Phe/Tyr ratio found in $\mathrm{HIV}$-1-infected individuals is a strong indication of $\mathrm{BH}_{4}$ deficiency, which is most probably due to oxidative loss of the oxidationsensitive cofactor in the state of chronic immune activation.

Changes in dopaminergic neurotransmission have been reported for HIV-infection in a number of studies. Decreased availability of dopamine in the central nervous system is correlated with low performance in neuropsychological functions and cannot be fully rescued by highly active ART (HAART) treatment (106, 107). Also, increased dopamine catabolism can contribute to dopamine deficiency. Monoamine oxidase activity was reported to 
be enhanced in specific brain regions of HIV-infected individuals and this was associated with HIV encephalitis (108).

Of note, the activity of the enzyme involved in serotonin synthesis, $\mathrm{TPH}$, is also dependent on $\mathrm{BH}_{4}$ levels, suggesting that potential decreases in $\mathrm{BH}_{4}$ during HIV infection may contribute to the losses in serotonin levels as well as to the dysregulation of dopamine and other catecholamine neurotransmitters.

\section{Metabolomic and Proteomic Approaches for Biomarker Identification}

In recent times, a number of new metabolites with potential prognostic value have been identified in large-scale metabolome biofluid analysis of HIV patients (109). Importantly, several studies confirmed the changes in metabolism of the above-mentioned amino acids Trp, Phe, and Tyr.

By analyzing the profile of oral metabolites in HIV-infected individuals, including ART-experienced and ART-naïve patients, in comparison to healthy controls, Ghannoum et al. reported differences in levels of metabolites that belong to especially carbohydrate and amino acid metabolism (110). In this study, Trp, Phe, and Tyr, as well as several downstream metabolites of these amino acids, were found to be upregulated in HIV-infected individuals compared to controls. Both Trp and Phe concentrations were somewhat higher in the oral wash of therapy-naïve versus experienced individuals. In line with previous results (20), the Phe/Tyr ratio was a reliable marker for the monitoring of the immune status during infection in this study. Otherwise, while in the circulation Trp breakdown via the increased activity of IDO1 in immune cells is highly accelerated (21), the consistently elevated levels of Trp in the oral wash samples indicate distinct metabolic properties in the oral cavity (110). Oral wash is an interesting body fluid for non-invasive diagnostics, but serum or plasma may still represent a better alternative when investigating immunological parameters. Although less accessible, CSF, which is close to the site of inflammation and linked to the neuropathology, may be best suited for assessing the association of various molecules in particular with disease-associated central nervous system-related symptoms $(111,112)$. A recent study assessing CSF metabolites in HIV patients with neurocognitive impairments reported alterations in Kyn levels, however, only for patients not on ART (113). In the same study, Phe and Tyr metabolism associated metabolite sets were enriched in patients with cognitive impairments. Besides oxidative stress and mitochondrial function, metabolite profiles revealed aging-related pathways to be associated with neurocognitive deficits.

Using a proteomic approach, Laspiur et al. (114) identified several molecules that are uniquely present in CSF of HIV patients with cognitive impairments. Among those molecules were sodium oxide dismutase, an antioxidant enzyme that was previously reported to be upregulated in the brains of HIVdemented patients compared to control patients (98), and migration inhibitory factor-related protein 14 (also known as protein S100A9), which is involved in the regulation of inflammatory processes and the immune response (114). In addition, other proteome studies have identified potential markers that might be associated with neuronal damage and inflammation $(115,116)$.

\section{Therapeutics and Future Directions}

A number of in vitro and animal studies are currently evaluating the potential of targeting IDO to limit HIV infection in combination with antiretroviral treatment. Blockade of IDO by 1 -methyl-D-tryptophan reduced viral loads in the plasma and lymph nodes of SIV-infected macaques that were also treated with ART (117). Whether long-term treatment with this combination is well tolerated, and whether it is effective at preventing neuronal damage or depression and/or at facilitating immune reconstitution still needs to be investigated. In fact, in animal tumor models, IDO1 inhibition by chemical or genetic interventions has been associated with the (re)activation of therapeutically relevant anticancer immune responses (118). Similar effects could be beneficial for immunodeficient patients.

Other treatment options are also currently being investigated. In a pilot study involving treatment of four HIV infected patients, high dose nicotinamide was effective at increasing tryptophan concentrations of patients (119). In addition, Lebouche and colleagues are investigating the effects of combined ART and niacin treatment on neurocognition and immune status, as well as on lipid metabolism of HIV-infected patients (120).

Recently, the combination of fluconazole and paroxetine (FluPar) was suggested as a therapeutic strategy for the treatment/ prevention of neurological damage in HIV-infected patients. FluPar was shown to be protective against HIV gp120- and Tatmediated neurotoxicity in a macaque model of SIV infection. Also, treatment of patients with ART and paroxetine (or another SSRI) was proposed as adjunctive neuroprotective and neuroregenerative therapy to treat HIV-infected individuals (121).

The CCR5 inhibitor maraviroc was shown to inhibit CNS replication of SIV in infected macaques, as well as to lower monocyte and macrophage activation, and to exert neuroprotective effects (122). In a study with $15 \mathrm{HIV}$-infected individuals, this drug improved the neurocognitive performance of the patients (123).

In a recent study in Wistar rats, facilitated transport of efavirenz (a non-nucleoside reverse transcriptase inhibitor) across the blood-brain barrier using phenylalanine-anchored solid lipid nanoparticles was shown to improve bioavailability and maintain therapeutic levels in the brain for an extended period of time, probably enabling a significant eradication of the viral load (124).

However, as discussed in Section "Vitamin Status, Nutrition, and Inflammatory Stress," nutritional interventions supporting drug therapy might be a first and very effective step to personalized treatment with less side effects.

\section{Conclusion}

A major issue in the treatment of HIV/AIDS is the development of neurocognitive disturbances in individuals despite effective therapy, although symptoms develop in a milder form or with changed dynamics $(4,10,125)$. Several factors are suggested to be involved in the progression of HAND, including the inability of drugs to effectively cross the blood-brain barrier, the development 
of resistant viral strains, and neuronal toxicity induced by viral proteins or chronic inflammation.

Elevations in Phe/Tyr, Kyn/Trp, and neopterin levels were shown in patients with HIV-1 infection, with the levels of neopterin in HIV-1 seropositives providing predictive information for the progression of AIDS and survival $(20,23)$. The cellular immune system is activated during HIV-1 infection, which leads to the activation of IFN- $\gamma$-dependent pathways including neopterin production via GCHI and tryptophan catabolism via IDO1. The concentrations of these biomarkers have been found to be altered in mental disorders and in disease-associated mood disturbances, and to correlate with depressive symptoms, especially, in long-lasting chronic diseases $(31,126)$. Of note, higher neopterin and Kyn/Trp were demonstrated in patients with HIV-infection (34) and accelerated Trp breakdown was shown to correlate with neuropsychiatric symptoms in HIV-patients (21, 33). In addition, chronic immune activation correlates with the decreased turnover of Phe (19), which has also been reported in HIV infection (20). Tyr and Trp are important precursors of serotonin and the dopaminergic neurotransmitters. The increased production of neopterin at the expense of $\mathrm{BH}_{4}$ and the lability of $\mathrm{BH}_{4}$ molecule under oxidative stress conditions (92) provides a rational link for the decreased activity of TPH and PAH enzymes, which use $\mathrm{BH}_{4}$ as a cofactor.

Recent metabolome and proteome analyses of changes occurring in the course of HIV-1 infection and therapy could confirm the dysregulation of amino acid metabolism and identify further pathways and molecules involved in HIV-associated neuroinflammation.

Numerous studies have reported an association between changes in immunologic parameters, such as the impaired activities of

\section{References}

1. Price RW, Brew B, Sidtis J, Rosanblum M, Scheck AC, Cleary P. The brain in AIDS: central nervous system HIV-1 infection and AIDS dementia complex. Science (1988) 239(4840):586-92. doi:10.1126/science.3277272

2. Antinori A, Arendt G, Becker JT, Brew BJ, Byrd DA, Cherner M, et al. Updated research nosology for HIV-associated neurocognitive disorders. Neurology (2007) 69:1789-99. doi:10.1212/01.WNL.0000287431.88658.8b

3. Price RW, Yiannoutsos CT, Clifford DB, Zaborski L, Tselis A, Sidtis JJ, et al. Neurological outcomes in late HIV infection: adverse impact of neurological impairment on survival and protective effect of antiviral therapy. AIDS clinical trial group and neurological AIDS research consortium study team. AIDS (1999) 13:1677-85. doi:10.1097/00002030-199909100-00011

4. Woods SP, Moore DJ, Weber E, Grant I. Cognitive neuropsychology of HIVassociated neurocognitive disorders. Neuropsychol Rev (2009) 19:152-68. doi:10.1007/s11065-009-9102-5

5. Gold JA, Grill M, Peterson J, Pilcher C, Lee E, Hecht FM, et al. Longitudinal characterization of depression and mood states beginning in primary HIV infection. AIDS Behav (2014) 18:1124-32. doi:10.1007/ s10461-013-0688-5

6. Gibbie T, Mijch A, Ellen S, Hoy J, Hutchison C, Wright E, et al. Depression and neurocognitive performance in individuals with HIV/AIDS: 2-year follow-up. HIV Med (2006) 7:112-21. doi:10.1111/j.1468-1293.2006.00350.x

7. Judd F, Komiti A, Chua P, Mijch A, Hoy J, Grech P, et al. Nature of depression in patients with HIV/AIDS. Aust N Z J Psychiatry (2005) 39:826-32. doi:10.1080/j.1440-1614.2005.01659.x

8. Judd FK, Mijch AM. Depressive symptoms in patients with HIV infection. Aust NZ J Psychiatry (1996) 30:104-9. doi:10.3109/00048679609076077 immunocompetent cells or increases in inflammatory mediators, and cytokine production with the activation of neuroendocrine pathways, altered neurotransmitter metabolism, and thus changes in behavior $(17,127)$. Current clinical parameters used to guide HIV-treatment are viral load and CD4 counts. These can probably deliver more diagnostic information when used in combination with an estimation of amino acid levels in patient serum. The targeted measurement of the well-established biomarkers neopterin, Kyn/Trp, and Phe/Tyr could be used to monitor the course of disease and judge the effectiveness of treatments in a reliable and relatively cost-effective manner. Although it was reported that ART could beneficially influence Phe/Tyr (20) and Kyn/Trp ratios (127), it still remains to be investigated whether a therapy-induced stabilization of amino acid levels also improves mood and quality of life in patients.

\section{Concluding Remarks}

Changes in mood status can be observed in the very early stages of HIV-1 infection (5) and, notwithstanding ART, HIV1 -infected individuals may develop cognitive impairment (10). The molecular changes underlying these infection-associated disturbances have not yet been characterized. Changes in the metabolism of the amino acid tryptophan and phenylalanine have been associated with HIV disease and were of predictive value. Both amino acids are precursors for neurotransmitter biosynthesis, providing a link to the development of disease-associated neurocognitive impairments. Therefore, monitoring amino acid metabolism in HIV-1-infected patients during ART could be useful in adapting personalized treatment regimens.

9. Everall I, Vaida F, Khanlou N, Lazzaretto D, Achim C, Letendre S, et al. Cliniconeuropathologic correlates of human immunodeficiency virus in the era of antiretroviral therapy. J Neurovirol (2009) 15:360-70. doi:10.3109/13550280903131915

10. Nightingale S, Winston A, Letendre S, Michael BD, McArthur JC, Khoo S, et al. Controversies in HIV-associated neurocognitive disorders. Lancet Neurol (2014) 13:1139-51. doi:10.1016/S1474-4422(14)70137-1

11. Burdo TH, Lackner A, Williams KC. Monocyte/macrophages and their role in HIV neuropathogenesis. Immunol Rev (2013) 254:102-13. doi:10.1111/ imr.12068

12. Hommes MJ, Romijn JA, Godfried MH, SchattenkerkJK, Buurman WA, EndertE, et al. Increased resting energy expenditure in human immunodeficiency virus-infected men. Metabolism (1990) 39:1186-90. doi:10.1016/0026-0495(90)90092-Q

13. Salomon J, de TP, Melchior JC. Body composition and nutritional parameters in HIV and AIDS patients. Clin Chem Lab Med (2002) 40:1329-33. doi:10.1515/ CCLM.2002.229

14. Vigouroux C, Gharakhanian S, Salhi Y, Nguyen TH, Chevenne D, Capeau J, et al Diabetes, insulin resistance and dyslipidaemia in lipodystrophic HIV-infected patients on highly active antiretroviral therapy (HAART). Diabetes Metab (1999) 25:225-32.

15. Villarroya F, Domingo P, Giralt M. Lipodystrophy associated with highly active anti-retroviral therapy for HIV infection: the adipocyte as a target of anti-retroviral-induced mitochondrial toxicity. Trends Pharmacol Sci (2005) 26:88-93. doi:10.1016/j.tips.2004.12.005

16. Hotamisligil GS. Inflammation and metabolic disorders. Nature (2006) 444:860-7. doi:10.1038/nature05485

17. Capuron L, Neurauter G, Musselman DL, Lawson DH, Nemeroff CB, Fuchs D, et al. Interferon-alpha-induced changes in tryptophan metabolism. relationship 
to depression and paroxetine treatment. Biol Psychiatry (2003) 54:906-14. doi:10.1016/S0006-3223(03)00173-2

18. Haroon E, Raison CL, Miller AH. Psychoneuroimmunology meets neuropsychopharmacology: translational implications of the impact of inflammation on behavior. Neuropsychopharmacology (2012) 37:137-62. doi:10.1038/ npp. 2011.205

19. Neurauter G, Schrocksnadel K, Scholl-Burgi S, Sperner-Unterweger B, Schubert C, Ledochowski M, et al. Chronic immune stimulation correlates with reduced phenylalanine turnover. Curr Drug Metab (2008) 9:622-7. doi:10.2174/138920008785821738

20. Zangerle R, Kurz K, Neurauter G, Kitchen M, Sarcletti M, Fuchs D. Increased blood phenylalanine to tyrosine ratio in HIV-1 infection and correction following effective antiretroviral therapy. Brain Behav Immun (2010) 24:403-8. doi:10.1016/j.bbi.2009.11.004

21. Fuchs D, Forsman A, Hagberg L, Larsson M, Norkrans G, Reibnegger G, et al. Immune activation and decreased tryptophan in patients with HIV-1 infection. J Interferon Res (1990) 10:599-603. doi:10.1089/jir.1990.10.599

22. Fuchs D, Hausen A, Reibnegger G, Werner ER, Werner-Felmayer G, Dierich MP, et al. Interferon-gamma concentrations are increased in sera from individuals infected with human immunodeficiency virus type 1. J Acquir Immune Defic Syndr (1989) 2:158-62.

23. Fuchs D, Jager H, Popescu M, Reibnegger G, Werner ER, Dierich MP, et al. Immune activation markers to predict AIDS and survival in HIV-1 seropositives. Immunol Lett (1990) 26:75-9. doi:10.1016/0165-2478(90)90178-S

24. Sperner-Unterweger B, Kohl C, Fuchs D. Immune changes and neurotransmitters: possible interactions in depression? Prog Neuropsychopharmacol Biol Psychiatry (2014) 48:268-76. doi:10.1016/j.pnpbp.2012.10.006

25. Wirleitner B, Reider D, Ebner S, Böck G, Widner B, Jaeger M, et al. Monocytederived dendritic cells release neopterin. J Leukoc Biol (2002) 72:1148-53.

26. Munn DH, Mellor AL. Indoleamine 2,3 dioxygenase and metabolic control of immune responses. Trends Immunol (2013) 34:137-43. doi:10.1016/j. it.2012.10.001

27. Mellor AL, Munn DH. Tryptophan catabolism and T-cell tolerance: immunosuppression by starvation? Immunol Today (1999) 20:469-73. doi:10.1016/ S0167-5699(99)01520-0

28. Sucher R, Fischler K, Oberhuber R, Kronberger I, Margreiter C, Ollinger R, et al. IDO and regulatory $\mathrm{T}$ cell support are critical for cytotoxic $\mathrm{T}$ lymphocyte-associated Ag-4 Ig-mediated long-term solid organ allograft survival. J Immunol (2012) 188:37-46. doi:10.4049/jimmunol.1002777

29. Munn DH. Indoleamine 2,3-dioxygenase, Tregs and cancer. Curr Med Chem (2011) 18:2240-6. doi:10.2174/092986711795656045

30. Widner B, Werner ER, Schennach H, Wachter H, Fuchs D. Simultaneous measurement of serum tryptophan and kynurenine by HPLC. Clin Chem (1997) 43:2424-6.

31. Schrocksnadel K, Wirleitner B, Winkler C, Fuchs D. Monitoring tryptophan metabolism in chronic immune activation. Clin Chim Acta (2006) 364:82-90. doi:10.1016/j.cca.2005.06.013

32. Fuchs D, Moller AA, Reibnegger G, Werner ER, Werner-Felmayer G, Dierich $\mathrm{MP}$, et al. Increased endogenous interferon-gamma and neopterin correlate with increased degradation of tryptophan in human immunodeficiency virus type 1 infection. Immunol Lett (1991) 28:207-11. doi:10.1016/0165-2478(91)90005-U

33. Fuchs D, Moller AA, Reibnegger G, Stockle E, Werner ER, Wachter H. Decreased serum tryptophan in patients with HIV-1 infection correlates with increased serum neopterin and with neurologic/psychiatric symptoms. J Acquir Immune Defic Syndr (1990) 3:873-6.

34. Schroecksnadel K, Sarcletti M, Winkler C, Mumelter B, Weiss G, Fuchs D, et al. Quality of life and immune activation in patients with HIV-infection. Brain Behav Immun (2008) 22:881-9. doi:10.1016/j.bbi.2007.12.011

35. Martinez P, Tsai AC, Muzoora C, Kembabazi A, Weiser SD, Huang Y, et al. Reversal of the Kynurenine pathway of tryptophan catabolism may improve depression in ART-treated HIV-infected Ugandans. J Acquir Immune Defic Syndr (2014) 65:456-62. doi:10.1097/QAI.0000000000000062

36. Quintana FJ, Basso AS, Iglesias AH, Korn T, Farez MF, Bettelli E, et al. Control of $\mathrm{T}(\mathrm{reg})$ and $\mathrm{T}(\mathrm{H}) 17$ cell differentiation by the aryl hydrocarbon receptor. Nature (2008) 453:65-71. doi:10.1038/nature06880

37. Bessede A, Gargaro M, Pallotta MT, Matino D, Servillo G, Brunacci C, et al. Aryl hydrocarbon receptor control of a disease tolerance defence pathway. Nature (2014) 511:184-90. doi:10.1038/nature13323
38. Fallarino F, Grohmann U, Vacca C, Orabona C, Spreca A, Fioretti MC, et al. T cell apoptosis by kynurenines. Adv Exp Med Biol (2003) 527:183-90. doi:10.1007/978-1-4615-0135-0_21

39. Fallarino F, Grohmann U, Vacca C, Bianchi R, Orabona C, Spreca A, et al. T cell apoptosis by tryptophan catabolism. Cell Death Differ (2002) 9:1069-77. doi:10.1038/sj.cdd.4401073

40. Schwarcz R, Bruno JP, Muchowski PJ, Wu HQ. Kynurenines in the mammalian brain: when physiology meets pathology. Nat Rev Neurosci (2012) 13:465-77. doi:10.1038/nrn3257

41. Heyes MP, Jordan EK, Lee K, Saito K, Frank JA, Snoy PJ, et al. Relationship of neurologic status in macaques infected with the simian immunodeficiency virus to cerebrospinal fluid quinolinic acid and kynurenic acid. Brain Res (1992) 570:237-50. doi:10.1016/0006-8993(92)90587-Y

42. Klein C, Patte-Mensah C, Taleb O, Bourguignon JJ, Schmitt M, Bihel F, et al. The neuroprotector kynurenic acid increases neuronal cell survival through neprilysin induction. Neuropharmacology (2013) 70:254-60. doi:10.1016/j. neuropharm.2013.02.006

43. Sas K, Robotka H, Toldi J, Vecsei L. Mitochondria, metabolic disturbances, oxidative stress and the kynurenine system, with focus on neurodegenerative disorders. J Neurol Sci (2007) 257:221-39. doi:10.1016/j.jns.2007.01.033

44. Schwarcz R, Whetsell WO Jr, Mangano RM. Quinolinic acid: an endogenous metabolite that produces axon-sparing lesions in rat brain. Science (1983) 219:316-8. doi:10.1126/science.6849138

45. Kandanearatchi A, Brew BJ. The kynurenine pathway and quinolinic acid: pivotal roles in HIV associated neurocognitive disorders. FEBS J (2013) 279:1366-74. doi:10.1111/j.1742-4658.2012.08500.x

46. Whetsell WO Jr, Schwarcz R. Prolonged exposure to submicromolar concentrations of quinolinic acid causes excitotoxic damage in organotypic cultures of rat corticostriatal system. Neurosci Lett (1989) 97:271-5. doi:10.1016/0304-3940(89)90609-5

47. Achim CL, Heyes MP, Wiley CA. Quantitation of human immunodeficiency virus, immune activation factors, and quinolinic acid in AIDS brains. J Clin Invest (1993) 91:2769-75. doi:10.1172/JCI116518

48. Sei S, Saito K, Stewart SK, Crowley JS, Brouwers P, Kleiner DE, et al. Increased human immunodeficiency virus (HIV) type 1 DNA content and quinolinic acid concentration in brain tissues from patients with HIV encephalopathy. J Infect Dis (1995) 172:638-47. doi:10.1093/infdis/172.3.638

49. Heyes MP, Brew B, Martin A, Markey SP, Price RW, Bhalla RB, et al. Cerebrospinal fluid quinolinic acid concentrations are increased in acquired immune deficiency syndrome. Adv Exp Med Biol (1991) 294:687-90. doi:10.1007/978-1-46845952-4_94

50. Heyes MP, Brew BJ, Martin A, Price RW, Salazar AM, Sidtis JJ, et al. Quinolinic acid in cerebrospinal fluid and serum in HIV-1 infection: relationship to clinical and neurological status. Ann Neurol (1991) 29:202-9. doi:10.1002/ana. 410290215

51. Valle M, Price RW, Nilsson A, Heyes M, Verotta D. CSF quinolinic acid levels are determined by local HIV infection: cross-sectional analysis and modelling of dynamics following antiretroviral therapy. Brain (2004) 127:1047-60. doi:10.1093/brain/awh130

52. Smith DG, Guillemin GJ, Pemberton L, Kerr S, Nath A, Smythe GA, et al. Quinolinic acid is produced by macrophages stimulated by platelet activating factor, Nef and Tat. J Neurovirol (2001) 7:56-60. doi:10.1080/13550280130 0069692

53. O'Connor JC, Lawson MA, André C, Briley EM, Szegedi SS, Lestage J, et al. Induction of IDO by bacille Calmette-Guérin is responsible for development of murine depressive-like behavior. J Immunol (2009) 182:3202-12. doi:10.4049/ jimmunol.0802722

54. Lawson MA, Kelley KW, Dantzer R. Intracerebroventricular administration of HIV-1 Tat induces brain cytokine and indoleamine 2,3-dioxygenase expression: a possible mechanism for AIDS comorbid depression. Brain Behav Immun (2011) 25:1569-75. doi:10.1016/j.bbi.2011.05.006

55. Drewes JL, Meulendyke KA, Liao Z, Witwer KW, Gama L, Ubaida-Mohien C, et al. Quinolinic acid/tryptophan ratios predict neurological disease in SIVinfected macaques and remain elevated in the brain under cART. J Neurovirol (2015). doi:10.1007/s13365-015-0334-2

56. Kerr SJ, Armati PJ, Pemberton LA, Smythe G, Tattam B, Brew BJ. Kynurenine pathway inhibition reduces neurotoxicity of HIV-1-infected macrophages. Neurology (1997) 49:1671-81. doi:10.1212/WNL.49.6.1671 
57. Sardar AM, Bell JE, Reynolds GP. Increased concentrations of the neurotoxin 3-hydroxykynurenine in the frontal cortex of HIV-1-positive patients. J Neurochem (1995) 64(2):932-5. doi:10.1046/j.1471-4159.1995.64020932.x

58. Fukui S, Schwarcz R, Rapoport SI, Takada Y, Smith QR. Blood-brain barrier transport of kynurenines: implications for brain synthesis and metabolism. J Neurochem (1991) 56:2007-17. doi:10.1111/j.1471-4159.1991.tb03460.x

59. Saito K, Lackner A, Markey SP, Heyes MP. Cerebral cortex and lung indoleamine-2,3-dioxygenase activity is increased in type-D retrovirus infected macaques. Brain Res (1991) 540:353-6. doi:10.1016/0006-8993(91)90536-5

60. Fu X, Lawson MA, Kelley KW, Dantzer R. HIV-1 Tat activates indoleamine 2,3 dioxygenase in murine organotypic hippocampal slice cultures in a p38 mitogen-activated protein kinase-dependent manner. J Neuroinflammation (2011) 8:88. doi:10.1186/1742-2094-8-88

61. Wolf $\mathrm{H}$. The effect of hormones and vitamin $\mathrm{B} 6$ on urinary excretion of metabolites of the kynurenine pathway. Scand J Clin Lab Invest Suppl (1974) 136:1-186.

62. Sainio EL, Pulkki K, Young SN. L-tryptophan: biochemical, nutritional and pharmacological aspects. Amino Acids (1996) 10:21-47. doi:10.1007/BF00806091

63. Kumar AM, Berger JR, Eisdorfer C, Fernandez JB, Goodkin K, Kumar M. Cerebrospinal fluid 5-hydroxytryptamine and 5-hydroxyindoleacetic acid in HIV-1 infection. Neuropsychobiology (2001) 44:13-8. doi:10.1159/000054908

64. Capuron L, Gumnick JF, Musselman DL, Lawson DH, Reemsnyder A, Nemeroff CB, et al. Neurobehavioral effects of interferon-alpha in cancer patients: phenomenology and paroxetine responsiveness of symptom dimensions. Neuropsychopharmacology (2002) 26:643-52. doi:10.1016/ S0893-133X(01)00407-9

65. Meulendyke KA, Queen SE, Engle EL, Shirk EN, Liu J, Steiner JP, et al. Combination fluconazole/paroxetine treatment is neuroprotective despite ongoing neuroinflammation and viral replication in an SIV model of HIV neurological disease. J Neurovirol (2014) 20:591-602. doi:10.1007/ s13365-014-0283-1

66. Ances BM, Letendre SL, Alexander T, Ellis RJ. Role of psychiatric medications as adjunct therapy in the treatment of HIV associated neurocognitive disorders. Int Rev Psychiatry (2008) 20:89-93. doi:10.1080/09540260701877670

67. Hill L, Lee KC. Pharmacotherapy considerations in patients with HIV and psychiatric disorders: focus on antidepressants and antipsychotics. Ann Pharmacother (2013) 47:75-89. doi:10.1345/aph.1R343

68. Baldewicz TT, Brouwers P, Goodkin K, Kumar AM, Kumar M. Nutritional contributions to the CNS pathophysiology of HIV-1 infection and implications for treatment. CNS Spectr (2000) 5:61-72.

69. Shor-Posner G, Feaster D, Blaney NT, Rocca H, Mantero-Atienza E, Szapocznik J, et al. Impact of vitamin B6 status on psychological distress in a longitudinal study of HIV-1 infection. Int J Psychiatry Med (1994) 24:209-22. doi:10.2190/7VD8-DA67-8T9L-UCHL

70. Rios-Avila L, Nijhout HF, Reed MC, Sitren HS, Gregory JF III. A mathematical model of tryptophan metabolism via the kynurenine pathway provides insights into the effects of vitamin B-6 deficiency, tryptophan loading, and induction of tryptophan 2,3-dioxygenase on tryptophan metabolites. J Nutr (2013) 143:1509-19. doi:10.3945/jn.113.174599

71. Linkswiler H. Biochemical and physiological changes in vitamin B6 deficiency. Am J Clin Nutr (1967) 20:547-61.

72. Da Silva VR, Rios-Avila L, Lamers Y, Ralat MA, Midttun $\varnothing$, Quinlivan EP, et al. Metabolite profile analysis reveals functional effects of 28-day vitamin B-6 restriction on one-carbon metabolism and tryptophan catabolic pathways in healthy men and women. J Nutr (2013) 143:1719-27. doi:10.3945/jn.113.180588

73. Giardina G, Montioli R, Gianni S, Cellini B, Paiardini A, Voltattorni CB, et al. Open conformation of human DOPA decarboxylase reveals the mechanism of PLP addition to Group II decarboxylases. Proc Natl Acad Sci U S A (2011) 108:20514-9. doi:10.1073/pnas.1111456108

74. Fernstrom JD. Role of precursor availability in control of monoamine biosynthesis in brain. Physiol Rev (1983) 63:484-546.

75. Fernstrom JD, Wurtman RJ. Brain serotonin content: physiological regulation by plasma neutral amino acids. Science (1972) 178:414-6. doi:10.1126/ science.178.4059.414

76. Leathwood PD. Tryptophan availability and serotonin synthesis. Proc Nutr Soc (1987) 46:143-56. doi:10.1079/PNS19870018

77. Murray MF. Tryptophan depletion and HIV infection: a metabolic link to pathogenesis. Lancet Infect Dis (2003) 3:644-52. doi:10.1016/S1473-3099(03)00773-4
78. Frick B, Gruber B, Schroecksnadel K, Leblhuber F, Fuchs D. Homocysteine but not neopterin declines in demented patients on B vitamins. J Neural Transm (2006) 113:1815-9. doi:10.1007/s00702-006-0539-x

79. Murr C, Winklhofer-Roob BM, Schroecksnadel K, Maritschnegg M, Mangge H, Bohm BO, et al. Inverse association between serum concentrations of neopterin and antioxidants in patients with and without angiographic coronary artery disease. Atherosclerosis (2009) 202:543-9. doi:10.1016/j.atherosclerosis.2008.04.047

80. Hendricks MK, Eley B, Bourne LT. Nutrition and HIV/AIDS in infants and children in South Africa: implications for food-based dietary guidelines. Matern Child Nutr (2007) 3:322-33. doi:10.1111/j.1740-8709.2007.00110.x

81. Gershon MD, Tack J. The serotonin signaling system: from basic understanding to drug development for functional GI disorders. Gastroenterology (2007) 132:397-414. doi:10.1053/j.gastro.2006.11.002

82. Jenny M, Klieber M, Zaknun D, Schroecksnadel S, Kurz K, Ledochowski M, et al. In vitro testing for anti-inflammatory properties of compounds employing peripheral blood mononuclear cells freshly isolated from healthy donors. Inflamm Res (2011) 60:127-35. doi:10.1007/s00011-010-0244-y

83. Gostner J, Ciardi C, Becker K, Fuchs D, Sucher R. Immunoregulatory impact of food antioxidants. Curr Pharm Des (2014) 20:840-9. doi:10.2174/13816128 113199990047

84. Knox WE. The regulation of tryptophan pyrrolase activity by tryptophan. $A d v$ Enzyme Regul (1966) 4:287-97. doi:10.1016/0065-2571(66)90023-9

85. Knox WE, Piras MM, Tokuyama K. Induction of tryptophan pyrrolase in rat liver by physiological amounts of hydrocortisone and secreted glucocorticoids. Enzymol Biol Clin (Basel) (1966) 7:1-10.

86. Murr C, Widner B, Wirleitner B, Fuchs D. Neopterin as a marker for immune system activation. Curr Drug Metab (2002) 3:175-87. doi:10.2174/1389200024605082

87. Werner ER, Werner-Felmayer G, Fuchs D, Hausen A, Reibnegger G, Wachter $\mathrm{H}$. Relationships between pteridine synthesis and tryptophan degradation. $A d v$ Exp Med Biol (1991) 294:177-84. doi:10.1007/978-1-4899-2638-8_40

88. Kraft-Terry S, Gerena Y, Wojna V, Plaud-Valentin M, Rodriguez Y, Ciborowski $\mathrm{P}$, et al. Proteomic analyses of monocytes obtained from Hispanic women with HIV-associated dementia show depressed antioxidants. Proteomics Clin Appl (2010) 4:706-14. doi:10.1002/prca.201000010

89. Patel KB, Stratford MR, Wardman P, Everett SA. Oxidation of tetrahydrobiopterin by biological radicals and scavenging of the trihydrobiopterin radical by ascorbate. Free Radic Biol Med (2002) 32:203-11. doi:10.1016/S0891-5849(01)00777-8

90. Kuzkaya N, Weissmann N, Harrison DG, Dikalov S. Interactions of peroxynitrite, tetrahydrobiopterin, ascorbic acid, and thiols: implications for uncoupling endothelial nitric-oxide synthase. J Biol Chem (2003) 278:22546-54. doi:10.1074/ jbc.M302227200

91. Wachter H, Fuchs D, Hausen A, Reibnegger G, Werner ER. Neopterin as marker for activation of cellular immunity: immunologic basis and clinical application. Adv Clin Chem (1989) 27:81-141. doi:10.1016/S0065-2423(08)60182-1

92. Werner-Felmayer G, Golderer G, Werner ER. Tetrahydrobiopterin biosynthesis, utilization and pharmacological effects. Curr Drug Metab (2002) 3:159-73. doi:10.2174/1389200024605073

93. Werner ER, Werner-Felmayer G, Fuchs D, Hausen A, Reibnegger G, Yim JJ, et al. Tetrahydrobiopterin biosynthetic activities in human macrophages, fibroblasts, THP-1, and T 24 cells. GTP-cyclohydrolase I is stimulated by interferon-gamma, and 6-pyruvoyl tetrahydropterin synthase and sepiapterin reductase are constitutively present. J Biol Chem (1990) 265:3189-92.

94. Schneemann M, Schoedon G, Hofer S, Blau N, Guerrero L, Schaffner A. Nitric oxide synthase is not a constituent of the antimicrobial armature of human mononuclear phagocytes. J Infect Dis (1993) 167:1358-63. doi:10.1093/ infdis/167.6.1358

95. Zangerle R, Fuchs D, Reibnegger G, Werner-Felmayer G, Gallati H, Wachter H, et al. Serum nitrite plus nitrate in infection with human immunodeficiency virus type-1. Immunobiology (1995) 193:59-70. doi:10.1016/S0171-2985(11)80155-5

96. Groeneveld PH, Kroon FP, Nibbering PH, Bruisten SM, van Swieten P, van Furth R. Increased production of nitric oxide correlates with viral load and activation of mononuclear phagocytes in HIV-infected patients. Scand J Infect Dis (1996) 28:341-5. doi:10.3109/00365549609037916

97. Torre D, Ferrario G, Bonetta G, Speranza F, Zeroli C. Production of nitric oxide from peripheral blood mononuclear cells and polymorphonuclear leukocytes of patients with HIV-1 infection. AIDS (1995) 9:979-80. doi:10.1097/00002030-199508000-00027 
98. Boven LA, Gomes L, Hery C, Gray F, Verhoef J, Portegies P, et al. Increased peroxynitrite activity in AIDS dementia complex: implications for the neuropathogenesis of HIV-1 infection. J Immunol (1999) 162:4319-27.

99. López M, San Román J, Estrada V, Vispo E, Blanco F, Soriano V. Endothelial dysfunction in HIV infection - the role of circulating endothelial cells, microparticles, endothelial progenitor cells and macrophages. AIDS Rev (2012) 14:223-30.

100. Calò LA, Caielli P, Maiolino G, Rossi G. Arterial hypertension and cardiovascular risk in HIV-infected patients. J Cardiovasc Med (Hagerstown) (2013) 14:553-8. doi:10.2459/JCM.0b013e3283621f01

101. Thomas SR, Mohr D, Stocker R. Nitric oxide inhibits indoleamine 2,3-dioxygenase activity in interferon-gamma primed mononuclear phagocytes. J Biol Chem (1994) 269:14457-64.

102. Ploder M, Neurauter G, Spittler A, Schroecksnadel K, Roth E, Fuchs D. Serum phenylalanine in patients post trauma and with sepsis correlate to neopterin concentrations. Amino Acids (2008) 35:303-7. doi:10.1007/s00726-007-0625-x

103. Scholl-Buergi S, Neurauter G, Karall D, Fuchs D. Serum phenylalanine concentrations in patients post trauma and burn correlate to neopterin concentrations. J Inherit Metab Dis (2009) 32:587-8. doi:10.1007/s10545-009-9960-2

104. Capuron L, Schroecksnadel S, Féart C, Aubert A, Higueret D, Barberger-Gateau $\mathrm{P}$, et al. Chronic low-grade inflammation in elderly persons is associated with altered tryptophan and tyrosine metabolism: role in neuropsychiatric symptoms. Biol Psychiatry (2011) 70:175-82. doi:10.1016/j.biopsych.2010.12.006

105. Anderson DN, Wilkinson AM, Abou-Saleh MT, Blair JA. Recovery from depression after electroconvulsive therapy is accompanied by evidence of increased tetrahydrobiopterin-dependent hydroxylation. Acta Psychiatr Scand (1994) 90:10-3. doi:10.1111/j.1600-0447.1994.tb01547.x

106. Kumar AM, Fernandez JB, Singer EJ, Commins D, Waldrop-Valverde D, Ownby $\mathrm{RL}$, et al. Human immunodeficiency virus type 1 in the central nervous system leads to decreased dopamine in different regions of postmortem human brains. J Neurovirol (2009) 15:257-74. doi:10.1080/13550280902973952

107. Kumar AM, Ownby RL, Waldrop-Valverde D, Fernandez B, Kumar M. Human immunodeficiency virus infection in the CNS and decreased dopamine availability: relationship with neuropsychological performance. J Neurovirol (2011) 17(1):26-40. doi:10.1007/s13365-010-0003-4

108. Meulendyke KA, Ubaida-Mohien C, Drewes JL, Liao Z, Gama L, Witwer KW, et al. Elevated brain monoamine oxidase activity in SIV- and HIV-associated neurological disease. J Infect Dis (2014) 210:904-12. doi:10.1093/infdis/jiu194

109. Sitole LJ, Williams AA, Meyer D. Metabonomic analysis of HIV-infected biofluids. Mol Biosyst (2013) 9:18-28. doi:10.1039/C2MB25318F

110. Ghannoum MA, Mukherjee PK, Jurevic RJ, Retuerto M, Brown RE, Sikaroodi M, et al. Metabolomics reveals differential levels of oral metabolites in HIV-infected patients: toward novel diagnostic targets. OMICS (2013) 17:5-15. doi:10.1089/ omi.2011.0035

111. Pendyala G, Want EJ, Webb W, Siuzdak G, Fox HS. Biomarkers for neuroAIDS: the widening scope of metabolomics. JNeuroimmune Pharmacol (2007) 2:72-80. doi:10.1007/s11481-006-9041-3

112. Price RW, Peterson J, Fuchs D, Angel TE, Zetterberg H, Hagberg L, et al. Approach to cerebrospinal fluid (CSF) biomarker discovery and evaluation in HIV infection. J Neuroimmune Pharmacol (2013) 8(5):1147-58. doi:10.1007/ s11481-013-9491-3

113. Cassol E, Misra V, Dutta A, Morgello S, Gabuzda D. Cerebrospinal fluid metabolomics reveals altered waste clearance and accelerated aging in HIV patients with neurocognitive impairment. AIDS (2014) 28:1579-91. doi:10.1097/ QAD.0000000000000303

114. Laspiur JP, Anderson ER, Ciborowski P, Wojna V, Rozek W, Duan F, et al. CSF proteomic fingerprints for HIV-associated cognitive impairment. J Neuroimmunol (2007) 192:157-70. doi:10.1016/j.jneuroim.2007.08.004
115. Gelman BB, Nguyen TP. Synaptic proteins linked to HIV-1 infection and immunoproteasome induction: proteomic analysis of human synaptosomes. J Neuroimmune Pharmacol (2010) 5:92-102. doi:10.1007/s11481-009-9168-0

116. Pendyala G, Fox HS. Proteomic and metabolomic strategies to investigate HIVassociated neurocognitive disorders. Genome Med (2010) 2:22. doi:10.1186/ gm143

117. Boasso A, Vaccari M, Fuchs D, Hardy AW, Tsai WP, Tryniszewska E, et al. Combined effect of antiretroviral therapy and blockade of IDO in SIV-infected rhesus macaques. JImmunol (2009) 182:4313-20. doi:10.4049/jimmunol.0803314

118. Vacchelli E, Aranda F, Eggermont A, Sautès-Fridman C, Tartour E, Kennedy EP, et al. Trial watch: IDO inhibitors in cancer therapy. Oncoimmunology (2014) 15:e957994. doi:10.4161/21624011.2014.957994

119. Murray MF, Langan M, MacGregor RR. Increased plasma tryptophan in HIVinfected patients treated with pharmacologic doses of nicotinamide. Nutrition (2001) 17:654-6. doi:10.1016/S0899-9007(01)00568-8

120. Lebouché B, Jenabian MA, Singer J, Graziani GM, Engler K, Trottier B, et al. The role of extended-release niacin on immune activation and neurocognition in HIV-infected patients treated with antiretroviral therapy - CTN PT006: study protocol for a randomized controlled trial. Trials (2014) 15:390. doi:10.1186/1745-6215-15-390

121. Steiner JP, Bachani M, Wolfson-Stofko B, Lee MH, Wang T, Li G, et al. Interaction of paroxetine with mitochondrial proteins mediates neuroprotection. Neurotherapeutics (2015) 12:200-16. doi:10.1007/s13311-014-0315-9

122. Kelly KM, Beck SE, Metcalf Pate KA, Queen SE, Dorsey JL, Adams RJ, et al. Neuroprotective maraviroc monotherapy in simian immunodeficiency virus-infected macaques: reduced replicating and latent SIV in the brain. AIDS (2013) 27:F21-8. doi:10.1097/QAD.0000000000000074

123. Ndhlovu LC, Umaki T, Chew GM, Chow DC, Agsalda M, Kallianpur KJ, et al. Treatment intensification with maraviroc (CCR5 antagonist) leads to declines in CD16-expressing monocytes in cART-suppressed chronic HIV-infected subjects and is associated with improvements in neurocognitive test performance: implications for HIV-associated neurocognitive disease (HAND). J Neurovirol (2014) 20:571-82. doi:10.1007/s13365-014-0279-x

124. Vyas A, Jain A, Hurkat P, Jain A, Jain SK. Targeting of AIDS related encephalopathy using phenylalanine anchored lipidic nanocarrier. Colloids SurfB Biointerfaces (2015) 131:155-61. doi:10.1016/j.colsurfb.2015.04.049

125. Cysique LA, Brew BJ. Neuropsychological functioning and antiretroviral treatment in HIV/AIDS: a review. Neuropsychol Rev (2009) 19:169-85. doi:10.1007/ s11065-009-9092-3

126. Widner B, Laich A, Sperner-Unterweger B, Ledochowski M, Fuchs D. Neopterin production, tryptophan degradation, and mental depression - what is the link? Brain Behav Immun (2002) 16:590-5. doi:10.1016/S0889-1591(02)00006-5

127. Zangerle R, Widner B, Quirchmair G, Neurauter G, Sarcletti M, Fuchs D. Effective antiretroviral therapy reduces degradation of tryptophan in patients with HIV-1 infection. Clin Immunol (2002) 104:242-7. doi:10.1006/clim.2002.5231

Conflict of Interest Statement: The authors declare that the research was conducted in the absence of any commercial or financial relationships that could be construed as a potential conflict of interest.

Copyright (c) 2015 Gostner, Becker, Kurz and Fuchs. This is an open-access article distributed under the terms of the Creative Commons Attribution License (CC BY). The use, distribution or reproduction in other forums is permitted, provided the original author(s) or licensor are credited and that the original publication in this journal is cited, in accordance with accepted academic practice. No use, distribution or reproduction is permitted which does not comply with these terms. 